\title{
RWPS: A Low Computation Routing Algorithm for Sensor Networks
}

\author{
Pierpaolo Bergamo, Daniela Maniezzo, Gianluca Mazzini \\ Engineering Dept., University of Ferrara, Italy \\ Mario Gerla \\ Computer Science Dept., UCLA, Los Angeles, CA, USA
}

\begin{abstract}
The recent interest in sensor networks has led to a number of routing schemes that use the limited resources available at sensor nodes more efficiently. These schemes typically try to find the minimum energy path to optimize the power consumption at a node. In this paper we propose an energy-efficient routing algorithm designed for sensor networks where an end-user wants to monitor remotely the environment. In such a situation, the data collected by each sensor must be sent to a central base station. We develop the Remote Watching Power Saving (RWPS) communication system, which involves routing and MAC layers, with the purpose of maximizing the lifetime of the sensor network distributing semi-randomly the load in the network and minimizing the routing signaling traffic.
\end{abstract}

\section{Introduction}

Recent advances in microelectronic and embedded technology and wireless communication have enabled the development of low-cost, low-power, multifunctional sensor nodes [1]. Sensor nodes are autonomous devices equipped with heavily integrated sensing, processing, and communication capabilities. When these nodes are networked together in ad hoc mode, i.e. without a coordination point for the wireless transmission, they form a sensor network. Generally, the nodes achieve data via their sensors, process it locally and forward the information to a data sink such as a central base station. Due to the limited transmission range of nodes, the forwarding mechanism involves paths across other nodes (relaying).

One of the most important problems in ad hoc networks is energy consumption, since wireless devices often run on batteries which have limited lifetime. Thus, key point for the effectiveness of the network is saving the energy. Every single layer should be optimized from the energy consumption point of view. For example, the physical layer should consume as few battery energy as possible; the link layer should be able to handle low power states and the network layer should implement an efficient routing protocol. The choice of a routing protocol determines somehow the frequency with which a node performs operations like receiving a packet and forwarding it.

Many researchers have addressed these issues. Havinga et al. [2] give an overview of the design process of low power devices and one of the strength points of the BlueTooth (BT) standard is the low power consumed by every single device. To reach this goal, the standardization organizations have defined different energy saving modes for the BT devices [3].

In pure sensors networks the energy efficiency is even more crucial due to the scale of typical networks. As a matter of facts, sensor networks are often thought for monitoring large geographical areas with a number of devices which can be enormously more than the number of devices in other ad hoc networks, like the IEEE802.11 [4] based ones. In this scenario, managing the available energy in a wise manner is one of the key problems. The idea is to limit the procedure of recharging the sensors which can be time and money consuming, due to the dimensions of the networks, and in some cases it is even not possible.

Many new metrics based on the consumed energy have been proposed for routing in wireless environment. In general the objective of these routing schemes is to increase the lifetime of each mobile node, increasing network lifetime as well [5]. In [6] the authors propose MTPR (Minimum Total Transmission Power Routing), a routing mechanism aiming to minimize the total power used to transmit a packet. MTPR gives higher priority to longer routes in terms of number of hops and its main drawback is that it can bring to high end-to-end delay. Moreover, since it does not consider the residual amount of energy in a node, it could bring to network partitioning. Given a certain topology, a set of nodes can be defined whose removal cause network partition [5]. To overcome this problem Li et al. proposed to use a metric to optimize the residual power of the overall network [7]. One problem which can be encountered in devising a routing mechanism for wireless networks is the partitioning due to the expiration of battery of some relay nodes. Routing solutions which consider the maximum fraction of remaining network energy in the optimal route computation have been devised to overcome this partitioning problem [7]. One drawback of this approach is the routing signaling packets which must be exchanged within the network. Such overhead can be very high in highly populated networks, like the sensors ones, and can jeopardize the gain of the algorithm itself.

Some authors consider to integrate routing and MAC layers in order to achieve a high energy reduction. One of the most common ways is to consider a power control, which should be implemented in a distributed fashion [8]. Very few works consider to realize a MAC protocol intrinsically implemented with 
routing capabilities. The main focus of this paper is to design an energy-efficient communication protocol, called RWPS (Remote Watching Power Saving), which involves both MAC and routing protocols. In our communication system, these two layers are deeply integrated. The routing protocol is power-aware: it tries to reduce the energy consumption hop by hop, basically without any periodic information exchanging, as it happens in proactive protocols. The routing layer does not require any path discovery procedure, as in the reactive protocols, because it is binded to the access mechanism. The MAC protocol is a time-slotted random access. Each operation is thought to be energy-efficient. On the other hand, the delivery time is not optimized. Nevertheless, we argue that in a environmental monitoring sensor network, the delivery time is not a strict requirement, since it makes no difference to the end-user to receive the interesting data with a delay of few milliseconds.

The paper is structured as follows. In Section 2 we give the details of RWPS protocol. In Section 3, the simulation platform is described. In Section 4, some results are presented. Finally, in Section 5, conclusions and future works are discussed.

\section{The Network and the Protocol}

\subsection{Network Description}

A realistic proposal for a sensor network considers to have the nodes switched off for a large percentage of time. If some event, such as the sudden increasing of the temperature or an activation particular audio frequency, shall be captured by the sensor network, a reasonable topology is dense, so that more than one sensor is able to capture the same event. These scheme is suitable for sensor networks for at least two reasons:

- the most part of the sensors can be switched off for a large percentage of time, reducing the consumption of the battery,

- the network connectivity is guaranteed even if some node is not perfectly working or its battery is low.

Thus, we consider a network in which a node is generally switched off and it switches on (randomly or with a fixed pattern) with a certain value of duty-cycle activity. Obviously, the node could stay switched on if the protocol requires it.

As we require a slotted time system, the node is supposed to keep awake for a time slot o multiple of a time slot.

The data packet is supposed to be exactly one time slot of length. This requirement is not so strict, if a sensor network for environment monitoring is considered. Due to the short life time of the batteries of sensors, a network in which nodes transmit a huge amount of data has no sense.

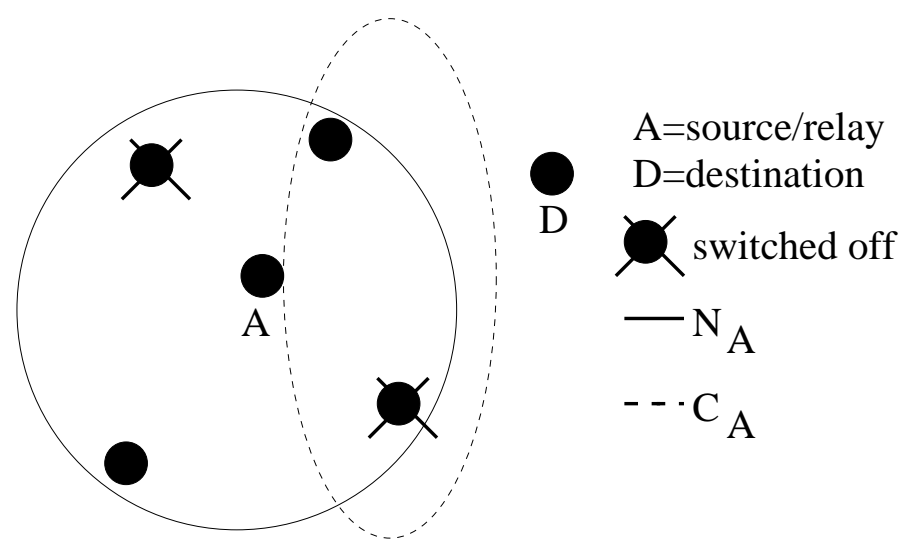

Figure 1: Definitions

\subsection{Assumptions}

We consider a network in which the sensors are synchronized, i.e., they can use a precise "common" timer to synchronize their operations. Realize such a system could be complex, but it cannot be done off-line before the deployment of the network. If the system requires to be synchronized once the network is deployed, the idea exploited in an interesting work made by J. Elson et al. [9] can be used, even in multihop scenarios.

Moreover, we consider that the nodes are manually deployed, which means that the sensors can be programmed with the information of their relative position on the network area. To do that, this information could be directly mapped as the MAC address of the node (in relative coordinates, the first half part of the protocol could represent the position on abscissa and the second one the position on ordinate). So, upon the reception of a packet, the receiving node knows its relative position with respect to the transmitting node. As an alternative, the nodes could be provided of autolocalization systems such as GPS embeddedly, even if this requirement is very hard to satisfy. The network is implicitly considered steady-state and the propagation rules symmetric (if node $a$ hears node $B$, also $B$ hears $A$ ).

Collisions of packets transmitted during the same time slot are considered lost, unless the capture effect system in the transceiver is able to recover one of them.

\subsection{Definitions}

Given a node $A$ (refer to Figure 1$)$ :

- $N_{A}(t)$ is the set of the nodes which are in radio coverage with $A$ ( $A$ is not included);

- $O N(t)$ is the set of nodes which are awake at time $t$;

- $O N_{A}(t)=O N(t) \bigcap N_{A}(t)$, which means that $O N_{A}(t)$ is the neighboring subset of $A$ awake at time $T$; 
- $C_{A}(t)$ is the subset of $N_{A}(t)$ of the nodes which are closer to the destination of the current packet than $A$;

- $C O N_{A}(t)=O N_{A}(t) \bigcap C_{A}(t)$.

Since the network is supposed to be steady-state, $N_{A}(t)$ is a function of time because of the time-fluctuating channel conditions and because of a node can get broken or its battery can expire. Simplifying the model, we suppose $N_{A}(t)=N_{A}$ and $C_{A}(t)=C_{A}$, i.e., each node is supposed to live forever and the channel fluctuations do not affect the sets of nodes in radio range, which can be realize with a proper value of sensitivity of the radio transceiver.

The first assumption (the node is supposed to have a infinite amount of energy) does not make the model far from reality, because we are interested in scenarios in which we want to optimize, with a fine tuning, the performance of the whole network. We do not want, for the analysis done in this paper, to have nodes running out of energy. This assumption will be removed in future works, when different conditions will be considered.

\subsection{Neighbor Discovering}

We consider a sensor network with a communication system which is time slotted without any features of carrier sensing, in order to simplify the hardware complexity. Suppose that the node $A$ has a packet to send ( $A$ can be the generator of the traffic or a relay). It sends a Probe packet on the next time slot (which will be called $t_{s}$ ). The Probe packet includes this information:

- the position of the source node $A$,

- the position of the destination node $D$.

The goal of a Probe is to discover what nodes are awake and can be relay of the communication, i.e., which is the $O N_{A}\left(t_{s}\right)$ set. After the reception of the Probe packet, the nodes in $O N_{A}\left(t_{s}\right)$ can switch off. Only the nodes that are in the set $C O N_{A}\left(t_{s}\right)$ will switch on in a time slot which is uniformly distributed in the set $\left[t_{s+1}, t_{s+W}\right]$. This time interval is called "Contention Window". Nodes know if they belong to $C O N_{A}\left(t_{s}\right)$ set by using the information in the Probe packet. In the chosen slot, they will send a Ready packet, in which they inform $A$ to be ready. The Ready packet includes this information:

- the position of itself,

- its battery status.

After that, they will switch off again, up to $t_{s+W+1}$, in which they will wake up, ready to receive the packet.

Collisions of Ready packets could occur, due to the randomness of the choice of the slot and also because of the possible parallel transmissions in an ad hoc networks. In this case, simply $A$ will not know that two or more nodes are ready to relay the packet. This will affect the routing decision, but not the effectiveness of the protocol. Moreover, it should be remarked that the capture effect system could recover one of the collided packets.

If for some reason the current relay does not receive any Ready packet, simply it retries the process at time $t_{s+W+1}$. This is choice is because of two reasons. The first reason is that some nodes could be awake, ready to receive the packet. This happens if collisions on the Ready packets occurred. In this case, the possible receivers can simply respond to the new Probe from the same node as in the described algorithm, dropping the previous transmit session. The second reason is that it makes no sense to wait a random (or a fixed) time before retrying the transmission, since the other nodes wake up randomly or at least with a pattern that could let the receiver find at least one receiver with a high probability.

\subsection{Routing Decision}

At time $t_{s+W+1}, A$ knows the set $R_{A}\left(t_{s}\right)$ of possible nodes to use as receivers/relays. In general, because of the collisions, $R_{A}\left(t_{s}\right) \subseteq C O N_{A}\left(t_{s}\right)$. Let $r_{i} \in R_{A}\left(t_{s}\right), i=1 \ldots M$ be the generic node of the considered set. $A$ will chose the node $r_{j}$ as relay, if:

$$
\frac{E_{r_{j}}}{d\left(r_{j}, D\right)}=\max _{i} \frac{E_{r_{i}}}{d\left(r_{i}, D\right)}
$$

where $d(x, y)$ is the distance (in meters) between node $x$ and $y$, computed with the information within the packet.

\subsection{Destination is in Radio Coverage}

When the destination node $(D)$ is in radio coverage with the transmitter/relay, the receiver is supposed to be awake, since it is the collecting node and it is supposed to have infinite energy. Basically, the last relay can simply transmit the packet the time-slot after it receives it.

If the assumption is not satisfied and the recipient is a common node, the protocol behaves as follows. The transmitter can know if it is in radio range with the destination by a threshold fixed as a system parameter. Basically, if the distance between the last relay and the destination is smaller than $R_{T H}$, the relay tries to transmit the Data packet. D will answer with an acknowledge if it is awake, otherwise the relay will retry to send the packet. The other nodes simply will ignore those attempts. If the relay is in low-battery status, it could decide to perform the normal Probe request and deliver the packet to a neighbor of the destination and so the relay can go in sleep mode.

Obviously, the normal Probe request can be used also for the last hop, even though the probability to transmit the Probe in the slot in which the destination node is awake is a function that raises if the duty-cycle raises and/or if $W$ decreases (which means that the power consumption would be very high and/or the collisions in the Contention Windows could have a high rate). 


\section{Simulation Platform}

In order to find results of the proposed protocol, we built a simulation platform. The software is able to simulate a generic number of nodes placed uniformly or as a grid network in a rectangular area. Each node can have different transmit power levels and a different initial battery status. Since it concerns with sensor networks for environment monitoring, mobility is not considered. The generation process is stochastic; $f$ a node can be a source during a certain slot, it has a random uniform probability to have a new packet to send. The destination can be always the same during a simulation or it can change for each new generated packet. No generation patterns or correlations among nodes in terms of space or time are considered. No noisy effects or fading are implemented in the simulator.

\section{Simulation Results}

\subsection{Setup Parameters}

$\mathrm{Q}=900$ stationary nodes are uniformly distributed in a 30units $\mathrm{x}$ 30units square area (where unit is a distance unit). The probability of a node to be switched on is $P_{o n}$ and it is a simulation parameter. In this paper, we consider to have infinite battery energy. The transmit power is the same for all the nodes and it is a parameter of simulation. The transmission power is settable as the maximum transmission radius $\mathrm{R}$ (which is measured in distance units). The Contention Window (W) measured in time-slots is a simulation parameter. Energy is measured in energy units. Each node can be a traffic source, with a probability $\lambda=10^{-5}$ (a reasonable value in our situation). The destination is always placed on the corner of the simulation square. In order to obtain the worst case performance, the destination node has the same probability of the other nodes to be switched on. This makes the last hop very hard to be successful. Nevertheless, the system performs very well.

The simulation time is $T=10^{7}$ time slots. The results are an average on many simulations.

\subsection{Results}

In Figure 2, the percentage of delivered packets is shown. We can see that RWPS is able to deliver a very high percentage of packets. With a value of $W \geq 12$, the system is able to deliver more than $80 \%$ of the packets. Since in our scenario, more than one sensor is able to capture the event to be monitored, this is sufficient to be reasonably sure that the even is notified to the collecting point. We want to remark that this is a very pessimistic performance, because of the assumption on the collecting point. If we consider $W<12$, too many collisions of Ready packets occur.

The delay, measured as the difference of the generation time and the delivery time to the final recipient, is shown in Figure

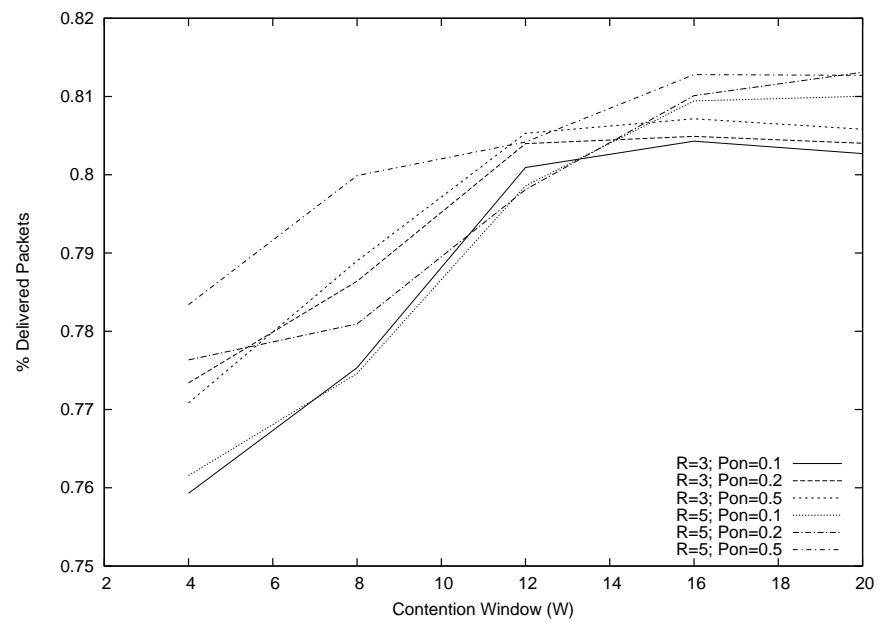

Figure 2: Percentage of delivered packets as a function of the Contention Window

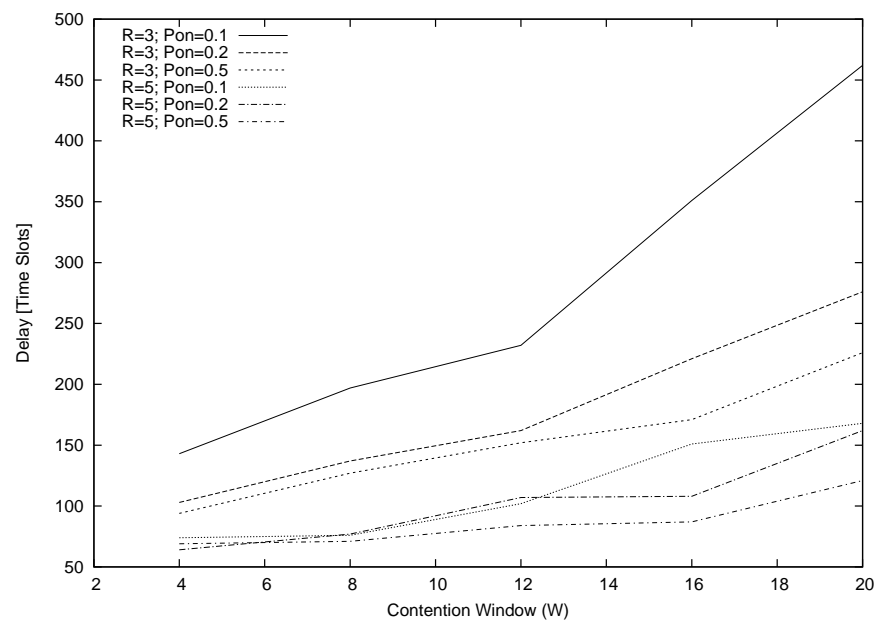

Figure 3: Delay as a function of Contention Window

3. It is still reasonably low with a Contention Window $W=12$ in all the considered conditions. The delay has a predictable behavior, since it is very close to a linear function of $R, P_{o n}$ and to an inverse function of $W$.

By looking at the energy results in Figure 4, it is possible to understand that the energy is basically a "flat line", slightly decreasing with the increasing values of $W$. Figure 4 reports the average energy spent among the nodes, considering transmission and reception. In any case, from such results it is possible to understand which is the proper battery level for the hardware, by normalizing the consumed energy with the simulation time.

\section{Conclusion}

In this paper, we propose a new communication system for sensor networks. The system, called RWPS (Remote Watching 


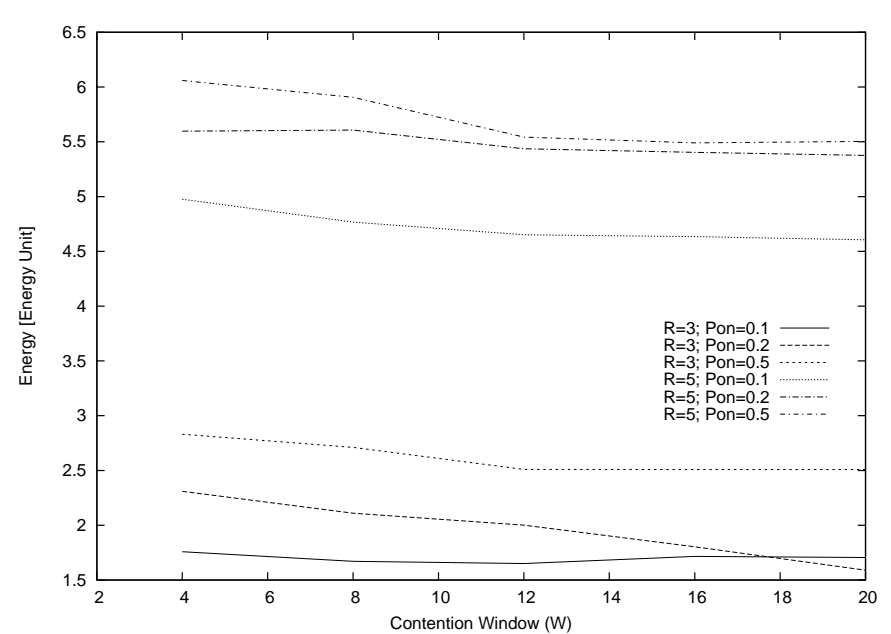

Figure 4: Energy as a function of Contention Window

Power Saving protocol) is to be used for environment monitoring. The typical scenario is a sensor network in which there is a collecting node, which is the sink of each communication. Nevertheless, the system is able to perform well also in a pure ad-hoc scenario, in which each node can send packets to a random destination.

RWPS can be considered a routing protocol deeply binded with the MAC layer in order to perform a very effective energysaving communication system. Simulation results in this paper show how what the requirements are to get the system working correctly. The requirement are reasonable.

In the future, we will develop an analytical model and we will stress the simulated network to understand the limits in terms of delay, throughput and life-time of the whole network.

\section{References}

[1] D. Estrin, R. Govindan, J. S. Heidemann, and S. Kumar, "Next century challenges: Scalable coordination in sensor networks", in Proc. 5th Ann. Intl. Conf. on Mobile Computing and Networking. Seattle, WA: ACM, Aug. 1999, pp. 263270 .

[2] P. J. M. Havinga, and G. J. M. Smith, "Design Techniques for Low-power Systems", Journal of Systems Architecture, Vol. 46, Iss. 1, 2000.

[3] "Specifications of the Bluetooth System" Core vol.1 v1.1, http: //www.bluetooth.com

[4] IEEE802.11 Working Group, "Wireless LAN Medium Access Control (MAC) and Physical Layer (PHY) Specification”, IEEE 802.11-97 Std., 1997.

[5] C. E. Jones, K. M. Sivalingam , P. Agrawal , J. C. Chen, "Survey of Energy Efficient Network Protocols for Wireless Networks", ACM Wireless Networks, September 2001.

[6] K. Scott and N. Bambos, "Routing and channel assignment for Low power transmission in PCS", Proc. ICUPC 1996, Oct. 2, 1996, pp. 498-502.

[7] J. Aslam, Q. Li, and D. Rus, "Distributed Energy-conserving Routing Protocols", In Proceedings of the Thirty-Sixth Annual Hawaii International Conference on System Sciences (HICSS), January 2003.

[8] P. Bergamo, D. Maniezzo, A. Giovanardi, G. Mazzini, M. Zorzi, "Distributed Power Control for Power-aware Energyefficient Routing in Ad Hoc Networks", European Wireless 2002, Florence, Italy, February 25-28, 2002, pp. 237-243.

[9] J. Elson, L. Girod, D. Estrin, "Fine-Grained Network Time Synchronization using Reference Broadcasts", in Proceedings of the Fifth Symposium on Operating Systems Design and Implementation (OSDI 2002), Boston, MA. December 2002. 